me in all the work done and in obtaining specimens; to the German Hospital which made it possible to secure nearly all the material, and to the Alexian Brothers' Hospital; and lastly, to Dr. J. A. Wesener who made all the chemic analyses, and offered suggestions that materially aided in making the work successful.

\section{CEREBROSPINAL PNEUMOCOCCUS INFECTION.}

REPORT OF A CASE.

BY BAYARD HOLMES, M. D.

CHICAGO.

There are many cases of rare clinic interest unreported, because the history is incomplete or because of loss of interest on the part of the observer, after the puzzle is solved by the post-mortem. Such cases are however very instructive to the reader, and the following is reported, in the hope of illustrating a rather obscure manifestation of pneumococcus infection:

On Monday, February 26 , I was called in consultation to see a girl 15 years old, who had been suffering during five weeks with a complex of symptoms resembling very closely the symptoms of relapsing fever. The sickness was initiated by a chill of great severity, followed by very high temperature and severe headache, and pain extending down the back and legs. The pulse was not as rapid as would ordinarily be expected with such a temperature. The temperature fluctuated about 104 and $10 \tilde{\mathrm{F}} \mathrm{F}$., for nine days, when there was a complete remission. The headache disappeared, the temperature and pulse became normal, and the young girl acted as a convalescent, ate and drank naturally, and slept well. This intermission lasted a little less than a week, when a second, but shorter, spell came on with a second chill. The temperature again rose to 104 , the pain in the head, the distress, and the high pyrexia returned. The pulse was again a little lower than we could ordinarily expect. This attack lasted seven days, when there was again a complete intermission, the temperature coming down to normal and with a cessation of all the other symptoms, as before. The third relapse occurred at the end of another week, with a chill and high tem. perature which lasted only two days, when there was an intermission of four days. A fourth relapse followed. It was during the second twenty-four hours of this relapse that I was called in and observed the girl. The patient's temperature was at this time 104 and her pulse 96 . The face and body showed some emaciation, the pupils were dilated and the child was vomiting at short intervals. The respirations were slightly accelerated. The little girl bagged for some relief, complained of her dreadful headache, grasped her head in her hands, and complained of the lightest touch over the scalp or face or arms or any part of the body. This hyperesthesia seemed to me most accentuated over the left side of the head, behind the ear. Her favorite position was curled up on her right side. The tongue was not noticeably coated, but the lips were chapped and about the nostrils there was some evidence of sordes. The heart and lungs and abdomen and extremities were perfectly negative on exam. ination, except during the first and second paroxysm of fever, when there was enlargement and tenderness of the liver and spleen. Careful search was made for an area of possible pneumonia, and the heart was carefully examined for endocarditis. The abdomen and pelvis were carefully examined for suppurative foci. All the joints were examined for evidence of infection. There was no tremor of the hands and no evidence of impairment of motion or of sensation -except the hyperesthesia mentioned. The hearing seemed to be equal in both ears and the sight seemed to be perfect in both eyes. There was no evidence of any perversion of taste and the only abnormal sensation was the general hyperesthesia and the severe headache, which was referred indefinitely to every part of the head.

The diagnosis seemed to rest between relapsing fever, malaria, typhoid fever, meningitis from middle ear disease, and meningitis from some other form of infection.

With the assistance of Dr. Hall, the blood was examined, and relapsing fever and malaria both excluded. The blood-count showed a slight leucocytosis, but otherwise was perfectly normal. As soon as this result had been determined a needle was introduced into the spinal canal at the junction of the third and fourth lumbar vertebræ, and the cerebrospinal fluid allowed to run off, drop by drop. Two agar tubes and two blood.serum plates were inoculated from this serum. In twenty-four hours they had every one shown a pure culture of a diplococcus, apparently the diplococcus of pneumonia. By this means the pathologic character of the disease was definitely determined. We had to deal with a case of pneumococcus infection of the cerebrospinal lymph-spaces. At this juncture, we determined on an experiment, and secured for a small fee, three ounces of blood from a healthy German man, 21 vears old, one week after a crisis from a true pneumococcus pneumonia. The blood was taken from the median vein, with all antiseptic precautions, into a sterilized flask, which was immediately put on ice and encouraged to coagulate rapidly by the addition of a few drops of calcium chlorid solution and inter. rupted heat and cold.

A sufficient amount of serum was at hand in twelve hours and hypodermic injection of this serum was begun; at first 60 drops, at each subsequent injection, at intervals of six hours, a double quantity. The effects of the injections seemed to be noticed at once, in a lowering of the temperature and general improvement. This continued for three days, when all the symptoms grew worse. There were repeated chills and an excessively high temperature, tremor of the extremities and apparently paralysis or an inability to co-ordinate the motions of the right leg and right arm. The right side of the face seemed to be less expressive. The patient continued to lie on the left side of the head, but turned on the right side of her body. The pupils were equally dilated, although no remedies had been administered which could produce such result. After this stupor had continued for twenty-four hours with a gradual aggravation of the symptoms; the ekin over the left mastoid seemed to be edematous. This was the first localizing sign which was incontrovertible, and I determined to ant on the assumption that the pneumococcus infection was due to a primary pneumococcus infection of the ear, and resulting pneumococcus meningitis and probably sinus thrombosis.

The patient's head was, therefore, shaved and prepared for operation. After shaving, the edema of the scalp over the mastoid was much plainer and a lymph 
gland as large as a bean was felt. The operation was begun with more confidence.

The mastoid cells were found edematous, and cul. tures were made from the contents. The skull above the root of the petrous portion was opened and the dura incised. A small amount of serum flowed out, and cultures were made from this serum. The sigmoid sinus was exposed: palpitation did not determine whether it was obliterated by thrombus or not. A small incision was therefore made in the sinus, from which venus blood poured out readily. The hemorrhage was stopped by means of a tampon, and the wound in the head dressed in an ordinary fashion. This operation produced little or no effect on the course of the disease.

In twelve hours the child died; a post-mortem was made eighteen hours later. On removing the dressing there was evidence of only a trifling amount of oozing. The skullcap came off, leaving the dura behind with no evidence of adhesions. On opening the dura the interlobular spaces seemed to be filled with a cloudy fibrinous exudate, and the pia was everywhere moderately injected. There was evidence of a slight amount of pachymeningitis along the middle portion of the longitudinal sinus on both sides. There was no evidence of meningitis at the base of the cerebrum. The base of the pons and medulla was covered with a yellowish firm pus-like exudate, which extended over two-thirds of the right hemisphere of the cerebellum, and one-third of the left. Cultures were made from the exudate and the adhesions near the longitudinal sinus. The dura was stripped off from the base of the skull, with no adhesions whatever npon the left side. The left sigmoid sinus and the lateral sinuses were free from thrombi. The right sigmoid sinus was occupied by a white thrombus. The dura was adherent to the posterior side of the petrous portion of the temporal bone, and a superficial examination led me to think that the petrosa was the seat of infection, but a more careful examination revealed its integrity.

\section{TREATMENT OF SYPHILIS*}

BY BACCELLI INTRAVENOUS INJECTIONS OF MERCURY. BY DUDLEY TAIT.

SURGEON OF FRENCH HOSPITAL. SAN FRANCISCO, CAL.

If therapeutics can be defined, "the knowledge of indications and the art of fulfilling them," it can be safely asserted and successfully maintained that the possession of so-called specific medications has been the signal of a retrograde step. No one will deny, for instance, the meager reflection accorded by the majority of medical men to the proper use and administration of such specific medicaments as mercury and quinin. The choice of the remedy being determined in advance, it would seem that nothing important re. mained to be considered; common sense is excluded by empiricism, and force of habit crowds out the allimportant question of indications. In no other morbid entity is such culpable disregard manifested as in syphilis. When questioned regarding the mode of treating this disease, the majority of practitioners invariably outline in two words, a simple, constant and uniform method; no reasons are given; no ques. tions asked. The diagnosis is the sole important factor. Thus, simplicity, which in therapeutics, medical

* Read at the Twenty-ninth Annual Meeting of the Medical Society of the State of California, April, 1899. and surgical, habitually marks progress, becomes a barrier to knowledge.

Convinced that syphilis presents a multiplicity of indications resulting from the variable degree of virulence of the infecting source, from the condition and resistance of the infected soil, and even from extrinsic factors, and believing that a vast number of the so-called incurable tertiary lesions ${ }^{1}$ can be avoided or cured, that the pathogenic rôle of syphilis in medullary and cerebral affections is of greater import than is generally conceded, that mercury is superior to the iodins under all circumstances, especially in malignant and tertiary lesions, the writer desires to call attention to a much neglected method of treatment, which, in his observation and experience of over two years, possesses, under certain well-defined conditions, many advantages over all other methods.

The intravenous method of treatment is based on the incontestable fact that mercury has no effect on the organism until it enters the blood. Furthermore, it has been demonstrated (Stassano, Metchnikoff) that the leucocytes are the exclusive vehicle and agent of absorption of the mercurial preparations.

The most practical solutions habitually used are the cyanid, $1 / 100$ and $1 / 200$, and the sublimate $1 / 500$ to 1/1000. The amount injected daily varies from 1 to 3 c.c.

The technic of these injections is both simple and safe; the skin over the flexor surface of the elbow having been cleansed, the arm is constricted by means of a rubber bandage, the ends of which are held by a clamp. Any prominent vein, any other region may be chosen, even the median basilic, for the needle can be controlled more easily than the lancet. The needle having been introduced obliquely, the constriction is removed by operating the clamp. With a little practice one will instantly know if the needle has pene. trated the vein. This may be ascertained by separa. ting the syringe from the needle and allowing a few drops of blood to escape. A better and safer plan suggested by the writer, is to use a medium-sized needle; in case the puncture is successful, within one or two seconds the blood will enter the syringe and color the mercurial solution. The patient should feel only the puncture of the skin, but if any fraction of the solution pass into the cellular tissue to the side or behind the vein, intense pain will be complained of immediately, followed by a painful induration which generally is absorbed after a period varying from two to three weeks. Extravasation of blood in the cellular tissue is a possible complication; it is most always due to the use of a needle of unnecessarily large cali. ber. The patient's arms should invariably be examined before the intravenous method is prescribed, for the cases are not infrequent ( 4 per cent. of the writer's cases) in which the veins can not be made sufficiently prominent to be punctured with safety. This difficulty is encountered particularly in women and in adipose patients. No dressing is required.

Danger of emboli and infection has been suggested as an objection to the intravenous medication, but upon theoretic grounds only. Experimentation demonstrates the contrary; no thrombus occurs in the vein in which an injection has been made. Hence, one can make a series of injections in the same vein; the writer has made as many as ten in the median. basilic vein without causing the slightest local or gen-

1 Excluding tabes, 50 per cent, of medullary affections are of syphilitic. origin. Gilles de la Tourette, Myćlites Syphilitiques. Paris, 1899. 